Kirt Areis E. Delovino, MD

William S. Lim, MD

Department of Otorhinolaryngology

Head and Neck Surgery

St. Luke's Medical Center
Correspondence: Dr. Kirt Areis E. Delovino

Department of Otorhinolaryngology

Head and Neck Surgery

St. Luke's Medical Center

279 E. Rodriguez Ave, Quezon City 1102

Philippines

Fax: (632) 7230101 local 5543

E-mail: edot_ii@yahoo.com

Reprints will not be available from the author.

The authors declared that this represents original material that is not being considered for publication or has not been published or accepted for publication elsewhere in full or in part, in print or electronic media; that the manuscript has been read and approved by all the authors, that the requirements for authorship have been met by each author, and that each author believes that the manuscript represents honest work.

Disclosures: The authors signed disclosures that there are no financial or other (including personal) relationships, intellectual passion, political or religious beliefs, and institutional affiliations that might lead to a conflict of interest.

Presented at the Instrumentation Paper Research Contest, Philippine Society of Otolaryngology Head and Neck Surgery Annual Convention, Crowne Plaza Galleria, Pasig City, Philippines, October 11, 2010

Editor's Note: The Philippine Journal of Otolaryngology Head and Neck Surgery publishes innovations in surgical technique and instrumentation that may be particularly relevant to developing-world settings, especially where they involve the use of recycled materials to produce a low-cost device, as in this case. Publication of these innovations in no way constitutes scientific endorsement of the device(s) described, by the publisher, editors and reviewers, and readers are reminded to exercise due caution in evaluating the device(s) described, and in their subsequent use.

\section{An Improvised Tracheotomy Speaking Valve}

\author{
ABSTRACT \\ Objective: To design and test an improvised tracheotomy speaking valve fabricated from \\ recycled parts of an anesthesia airway breathing circuit.
}

\section{Methods:}

Design: Surgical Instrumentation

Setting: Tertiary Private Hospital

Subjects: Speaking valves fabricated from discarded anesthesia breathing circuit parts were pilot-tested on three patients: one with vocal fold paralysis, another with a supraglottic mass and one post hemi-laryngectomy.

Results: The improvised tracheotomy speaking valve was inexpensive and relatively easy to assemble. All three patients tolerated speech well through the speaking valve and were pleased to reestablish their means of verbal communication. Maximum Phonation Time (MPT) averaged 8 seconds for all three subjects.

Conclusion: In our local setting, improving the quality of life of tracheotomized patients should be accessible to all, hence the value of an improvised speaking valve. It provides a more affordable means of restoring speech and because it is made from recycled materials, it is ecofriendly. Our improvised speaking valve is also a cheaper but viable alternative to more expensive commercially available ones. Clinical trials with standardized feedback questionnaires, multiobserver perceptual evaluation with a system such as the GRBAS and/or vocal acoustic measures in a speech laboratory should be made to assess long term use, efficiency and safety measures.

Keywords: tracheotomy, tracheostomy, speaking valve, speaking device

Tracheotomy is a common essential surgical procedure that establishes an alternative airway. The disadvantage of this often-lifesaving procedure is altered ability to verbally communicate effectively, which may lead to depression. Speech is an integral part of quality of life, and its loss results in psychological and emotional strain. Various devices have been developed over the years to improve speech in cognitive, tracheotomized patients. From aesthetic to functional accessories, the speaking tracheotomy tube has evolved to what we know now as the speaking valve. A tracheotomy speaking valve works by allowing inspired air to pass through it and the tracheotomy tube whilst directing exhaled air towards the glottis to provide pulmonary airflow for phonation. However, the high cost of these devices limits their availability to only a select few in a developing country like the Philippines. There is thus a need for an affordable yet durable alternative, without compromising pulmonary function and aesthetic value.

We designed and pilot-tested an improvised tracheotomy speaking valve fabricated from recycled parts of an anesthesia airway breathing circuit, as a cheaper substitute to commerciallyavailable speaking valves. 


\section{METHODS}

The primary materials used $(1,2)$ were taken from discarded anesthesia breathing circuits:

1. Endotracheal Tube external adaptor (Portex Tracheal Tube $7.5 \mathrm{~mm}$ Soft Seal Cuff Oral, Smiths Medical International Ltd., Brisbane, Australia)

2. Airway Breathing Circuit elbow connector (Anesthesia Breathing Circuit A10007183, Inspired Medical, Vincent Medical Manufacturing Co., Ltd., Kowloon, Hong Kong)

3. Transparency OHP Acetate Film Clear, A4 210mm x 297mm 100 micron (Star 360 Philippines Inc, Quezon City, Philippines)

4. Fine-toothed hacksaw blade (Eclipse All Hard Power Hacksaw Blades, Model: ECL.AE 353K Blade size $400 \times 32 \times 1.60 \mathrm{~mm} ; 16^{\prime \prime} \times 1$ $1 / 4^{\prime \prime} \times 0.06$ " teeth per inch: 10 (Neill Tools, Spear \& Jackson, Sheffield, England)

5. Multipurpose Cutter No. 760 (KW-Trio Company LTD, Taiwan)

6. Silicone Carbide Abrasive Sand Paper $320 \mathrm{C}$ (Crocodile Brand, Korea)

The anesthesia airway breathing circuit $(A B C)$ elbow connector has a universal inner diameter size of $15 \mathrm{~mm}$ which is commonly used with most endotracheal tubes (ETT) and tracheostomy tubes. Endotracheal tube external adaptors and tracheostomy tubes on the other hand, have standard outer diameters of $14 \mathrm{~mm}$ which fit snugly in place with $A B C$ elbow connectors.

With a fine-toothed saw, the $A B C$ elbow was cut just before the bend so that a plastic cylinder, open at both ends, was produced. (Figures 1-2) To hold a flap that could seal one end of this cylinder during breathing, a $2 \mathrm{~mm}$ thickness was then trimmed from the distal end of the ETT external adaptor to make a ring. (Figure 3) With a cutter, a circular flap with a small protruding tongue was then fashioned from a sheet of acetate. The flap was positioned with the tongue ready to be wedged between the ring and the cylinder. The ring was then inserted tightly with the acetate flap at the distal portion of the cylinder to hold the flap tongue in place and to serve as a stopper during exhalation. (Figures 4-5)

During inhalation the flap moves towards the patient allowing free flow of air and conversely, during exhalation, the acetate flap shuts off securely against the inner ETT ring creating unidirectional airflow. During exhalation, the air is directed towards the glottis to provide pressure for phonation. (Figures 6-7)

With informed consent, the device was then pilot-tested on three patients from the social service outpatient department of the St. Luke's Medical Center. (Figure 8) One patient had bilateral vocal fold paralysis, another had a supraglottic mass and the third was a post hemi-

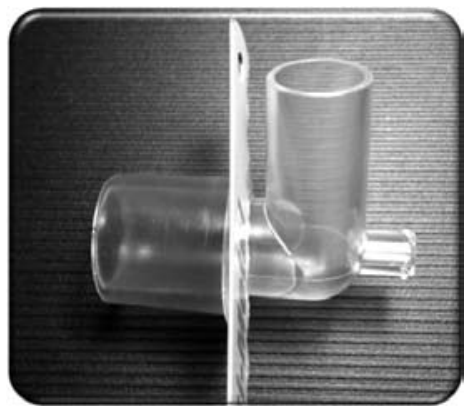

Figure 1. The Airway Breathing Circuit $(A B C)$ elbow was cut before the angle so that a cylinder is produced.

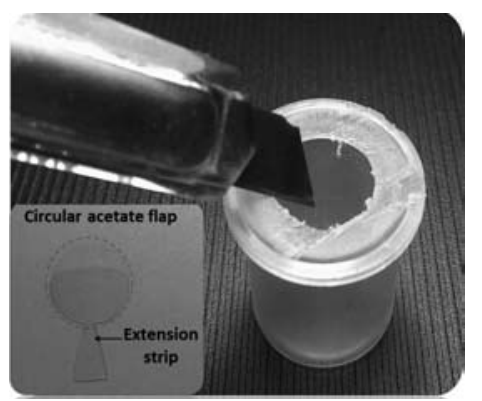

Figure 2. The rough edges of the cylinder were evened out using a multipurpose cutter and sand paper. Inset shows circular acetate flap with extension strip

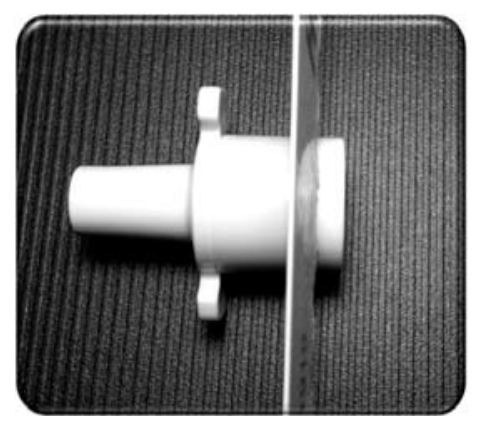

Figure 3. A $2 \mathrm{~mm}$-thick ring was tailored from the distal end of the Endotracheal Tube external adaptor

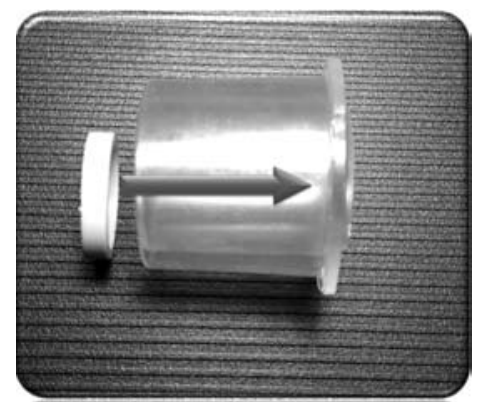

Figure 4. This ring was poised to fit snugly inside the $A B C$ cylinder at the distal end and serve as a "stopper" to hold the flap in position 


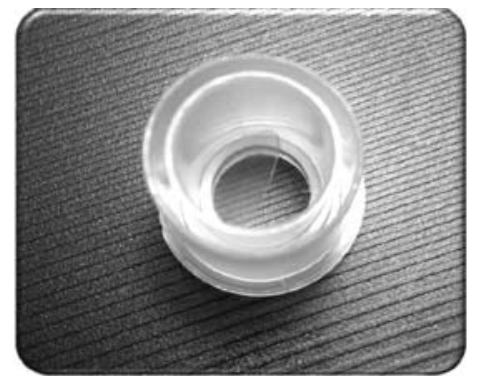

Figure 5. A circular acetate flap was positioned with the small tongue extension wedged between the cylinder and the ring

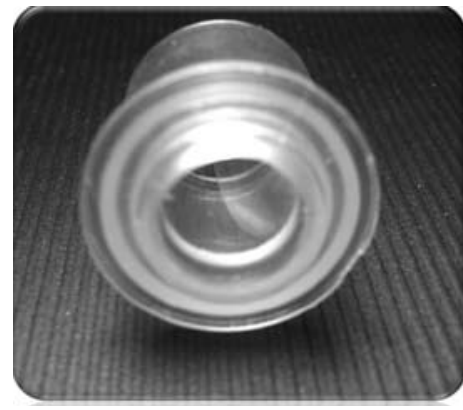

Figure 6. Finished product; the acetate flap moves freely with airflow, being wedged only by the small tongue

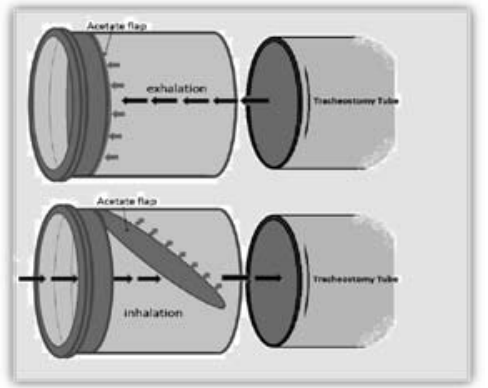

Figure 7. Mechanism of flap movement with airflow during inhalation and exhalation

laryngectomy patient, all of whom required long-term tracheotomy.

Using a fenestrated inner cannula and tracheotomy tube with inflated cuff, the subjects were seated upright and under normal breathing circumstances were asked to speak. We observed the quality of their voices and obtained feedback on the ease of use of the improvised speaking valve. We also obtained a three-trail average Maximum Phonation Time (MPT), the maximum length of time (in seconds) in which a person can sustain a vowel sound on one deep breath at a comfortable pitch and loudness, which is acceptable if greater than 7 seconds, in patients where glottic efficiency is poor. ${ }^{6}$
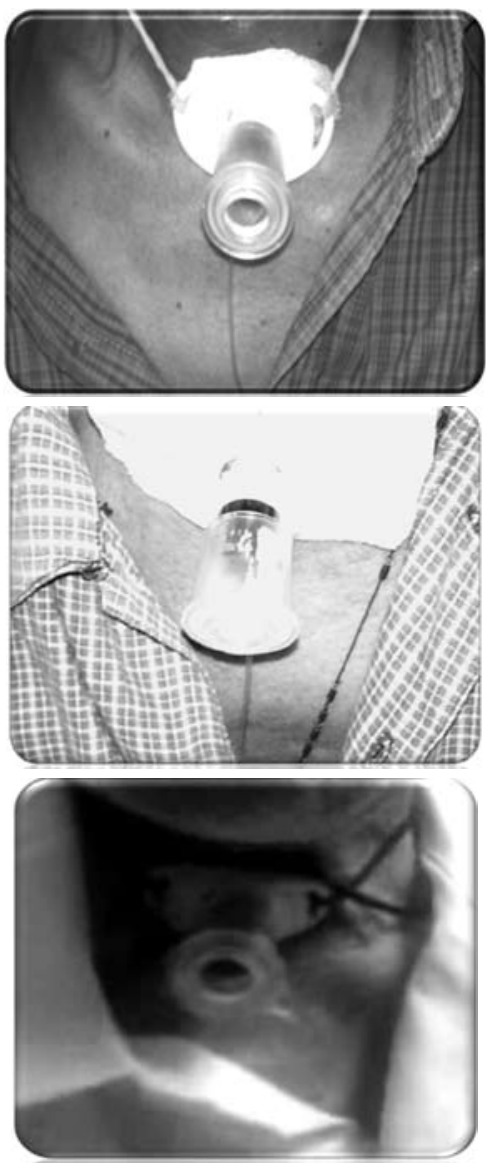

Figure 8. The device was pilot-tested on three patients from the social service out-patient department of our hospital

\section{RESULTS}

All three patients tolerated speech using the improvised tracheotomy speaking valve without difficulty of breathing. Although with a harsh and breathy quality, their voices were clearly understood. Our subjects verbalized that the device was easy to use. They also liked the fact that they did not have to manually occlude the tube anymore while speaking. One patient who previously utilized intermittent corking of the tracheotomy tube during speech claimed to have decreased breathing effort after using the device, because inspiration with corking required more effort to overcome upper airway resistance. Using our device made it easier and more comfortable for the patients to breathe freely without the need for manual occlusion of their tracheotomy tubes. It was easily adapted and did not require any special training or breathing exercises. Maximum Phonation Time (MPT) averaged 8 seconds in all three patients.

Even if we had used a new endotracheal tube external adaptor \& $A B C$ elbow connector, the production cost of the innovative speaking 
SURGICAL INNOVATIONS AND INSTRUMENTATION

Table 1. Cost of Production

\begin{tabular}{|l|c|}
\multicolumn{1}{|c|}{ Material } & Price \\
\hline Endotracheal Tube (External Adaptor) & P400* \\
\hline Airway Breathing Circuit (Elbow Connector) & P350* \\
\hline Transparency OHP Acetate Film & P 45 \\
\hline Fine-toothed hacksaw blade & P 25 \\
\hline Multipurpose Cutter & P 35 \\
\hline Abrasive Paper (Sandpaper) & P 15 \\
\hline TOTAL & P870 \\
\hline
\end{tabular}

*price based on brand new item

valve would have been a mere Php 870 (Table 1) - a great difference from commercially available speaking valves which range from PhP5,000 to 10,000 each depending on the brand and number of accessories.

\section{DISCUSSION}

Tracheotomies are performed for a variety of indications and maintained for different durations. When prolonged tracheotomy is necessary, it becomes disabling and adversely affects the patient's capability to effectively communicate verbally. This impacts on quality of life and may lead to psychosocial complications. In our study, the patients became accustomed without difficulty to the use of our "lowcost" improvised device. Furthermore, the patients expressed ease of use and comfort during phonation.

Voice production requires good pulmonary support. In spontaneously-breathing patients with tracheotomy tubes this is made possible by adding a valve that will allow air to pass through the larynx during phonation. The speaking valve directs the exhaled air through the upper airway allowing the patient to speak.

Although many patients may benefit from a speaking valve, there are also contraindications to its use. According to Hess ${ }^{3}$ speaking valves should only be used in fully conscious patients who are able to follow commands and attempt to communicate. Airway clearance should be assessed completely as secretions may obstruct airflow and compromise the airway. Upon application of the speaking valve, the patient's ability to breathe should be carefully assessed as many patients require long periods before acclimatization. In our subjects, the use of the improvised device was easily adopted. Neither further adjustments nor exercises were necessary to familiarize with its use. Although flap malfunction from adherence to the stopper due to tracheal secretions was theoretically possible, the patients were cautioned to immediately remove the device as a precautionary measure in such an eventuality.
During the assessment no adverse events were observed.

Commercially-available speaking valves are much more expensive, precluding their availability to financially-disadvantaged patients. Our improvised speaking valve provides an alternative to restoring speech without causing undue financial burden.

We were able to improvise a tracheotomy speaking valve devised from recycled materials which was functional and eco-friendly. The patients had their hands free to do other things while speaking. The device was easily adopted even without special preparations. Maximum phonation time averaged 8 seconds indicating efficiency of its use. Formal clinical trials are recommended to assess long term use, efficiency and safety issues especially regarding materials fatigue and maintenance procedures for daily care of the device. A standardized feedback questionnaire, and multi-observer perceptual evaluation with a system such as the GRBAS and/or vocal acoustic measures in a speech laboratory should be utilized in these trials.

\section{REFERENCES}

1. Elpern EH, Scott MG, Petro L, Ries MH. Pulmonary aspiration in mechanically ventilated patients with tracheostomies. Chest 1994 Feb; 105(2):563-566.

2. Suiter DM, McCullough GH, Powell PW. Effects of cuff deflation and one-way tracheostomy speaking valve placement on swallow physiology. Dysphagia 2003 Fall; 18(4):284-292.

3. Hess DR. Facilitating speech in the patient with a tracheostomy. Respir Care 2005 Apr; 50(4): 51925.

4. Stachler RJ, Hamlet SL, Choi J, Fleming S. Scintigraphic quantification of aspiration reduction with the Passy-Muir valve. Laryngoscope 1996 Feb;106 (2 Pt 1):231-4.

5. Elpern EH, Borkgren Okonek M, Bacon M, Gerstung C, Skrzynski M. Effect of the Passy-Muir tracheostomy speaking valve on pulmonary aspiration in adults. Heart Lung 2000 Jul-Aug; 29(4):287-293

6. Graham Williamson, MPT as a Measure of Vocal Function. Speech Therapy and Information Resources, 2008 [cited 2008 November 24] 22:11 Available from: http://www.speech-therapyinformation-and-resources.com/maximum-phonation-time.html 